\title{
Adsorption and characterization of MCPA on DDTMA- and raw-montmorillonite: Surface sites involved
}

\author{
Cintia C. Santiago, Mariela. A. Fernández, and Rosa M. Torres Sánchez \\ Technology Center of Mineral Resources and Ceramics, M. B. Gonnet, Argentina
}

\begin{abstract}
The 4-chloro-2-methylphenoxy acid (MCPA) is an herbicide widely used in agriculture, which generates a great concern about contamination of surface water and serious consequences for human health and the environment. In this work, the adsorption of MCPA on an Argentine montmorillonite (MMT) and its organo-montmorillonite product (OMMT) with different dodecyl trimethyl ammonium loading was investigated. MCPA adsorption on OMMT increases at least 3 times, with respect to the amount determined for MMT. X-ray diffraction and zeta potential analyses indicated the inner (interlayer) and outer surface participate as adsorption sites. Changes in surface electric charge and also interlayer expansion suggest that dimethyl amine (MCPA counterion) was also surface-adsorbed. The larger aggregates of OMMT, without and with MCPA, obtained compared to those of MMT samples, generate an improvement in the coagulation efficiency. This property, particularly after MCPA retention, allows an easier separation of the solids from the solution and enables a simple technological process application.
\end{abstract}

\section{ARTICLE HISTORY}

Received 28 August 2015

\section{KEYWORDS}

Adsorption; characterization; outer and inner surface sites; MCPA; organo-

montmorillonite

\section{Introduction}

Several treatments for pesticide removal from water have been studied with varying success, such as photocatalytic degradation, nanofiltration membranes, ozonation, aerobic degradation and adsorption. ${ }^{[1]}$ Among them, ozonation and adsorption have specifically been used to remove 4-chloro-2-methylphenoxyacetic acid (MCPA) from water. Due to the low cost of adsorption methods, also different adsorbent materials have been studied to address the purification of polluted water by acidic phenoxy herbicides: soil components, ${ }^{[2-6]}$ layered double hydroxides, ${ }^{[7-9]}$ metal oxides, ${ }^{[10]}$ resins, ${ }^{[11]}$ carbon, ${ }^{[12,13]}$ shales, ${ }^{[14]}$ etc.

Particularly, the use of raw clay minerals have revealed very low adsorption efficiency for MCPA, ${ }^{[15]}$ while the modification from hydrophilic to hydrophobic attained in organo-clays, increased its affinity for many pesticide molecules, among them anionic herbicides. ${ }^{[16-18]}$ These surface modifications, where electrostatic forces are also involved, also contribute to a better separation of the organo-clay systems from the solution. Then, as was found previously for bio-montmorillonite systems, ${ }^{[19]}$ the goal of a better isolation from the solution, after a remediation process, could improve the technological applications of the system.

Among the clays used to obtain organo-clays by cationic exchange with quaternary ammonium salts, montmorillonite (MMT) is one of the most widely employed. MMT belongs to smectite family clays, presenting a layered structure (2:1) that is formed by one octahedral alumina sheet sandwiched between two tetrahedral silica sheets. In the tetrahedral layer, Si atoms are partly substituted by $\mathrm{Al}$ atoms, while in the octahedral layer
$\mathrm{Al}$ atoms are replaced by cations with lower oxidation number $\left(\mathrm{Mg}^{+2}\right.$ or Fe $\left.{ }^{+2}\right)$. The negative charge surface originated by the isomorphic substitution is balanced by exchangeable cations (mainly $\mathrm{Na}^{+}$and $\mathrm{Ca}^{2+}$ ) located in the interlayer space, where also the organic cations located when organo-clays are obtained. ${ }^{[20]}$

The MCPA is a weakly acidic phenoxy herbicide extensively used to control broad-leaved weed growth in crops. It ranks among the 10 most important pesticides used in Europe. ${ }^{[21]}$ MCPA is insoluble in water and due to its anionic status; it is weakly retained by most of the soil components, so it remains dissolved in the soil solution with great concern for its increasing likelihood of run off and leaching to ground and surface waters. It is classified by the U.S. environmental protection Agency (EPA) as potential groundwater contaminant, with a $47.3 \mu \mathrm{g} \mathrm{L}^{-1}$ estimated environmental concentration for groundwater sources of drinking water for use without risk to human health. ${ }^{[22]}$

Particularly for MCPA remotion, the replacement of natural inorganic exchange cations in MMT with different organic cations such as L-carnitine, spermine, hexadimethrine, tyramine, dodecyl ammonium, N, N'di-dodecyl-N, N'tetramethyl diammonium diiodide, phenyl trimethyl ammonium, and hexadecyltrimethyl ammonium have been investigated with varying success, ${ }^{[15,17,23]}$ where not only the organic cations but also their exchanged percentage and the cation exchange capacity (CEC) of the clay played an important role. ${ }^{[15]}$

CONTACT Rosa M. Torres Sánchez rosats@cetmic.unlp.edu.ar, rosa.torres@gmail.com @ CETMIC (Centro de Tecnología de Recursos Minerales y Cerámica) CCT-La Plata. CIC BA. Camino Centenario y 506 (CC.49) (B1897ZCA), M. B. Gonnet, Argentina. 
In a series of previous papers, herbicides such as glyphosate (N-phosphonomethyl glycine) and diuron [3- (3, 4-diclhorophenyl)-1, 1-dimethylurease], and fungicides such as benzimidazole [2-(thiazol-4-yl)] were adsorbed on raw and thermally or mechanically treated MMT clay, where the importance of their outer and inner (interlayer) surface sites was revealed. ${ }^{[24]}$ Particularly, the glyphosate adsorption isotherms showed two well-defined step profiles, indicating its adsorption on the outer surface (related to aluminol and/or silanol groups) and on the interlayer space or inner surface (by a cationic exchange mechanism ${ }^{[25]}$ ). Two different techniques have proved to be skillful to follow the presence of surfactant or organic molecules in the inner and outer surface of the MMT. X-ray diffraction (XRD) and Zeta potential measurements indicated, the arrangement of surfactants in the interlayer space by measuring the shift of basal spacing reflection $(001),{ }^{[20,26]}$ and revealing their adsorption on the outer surface, ${ }^{[24]}$ respectively.

A precise knowledge of the MCPA adsorption sites involved are need to develop future research, e.g., to achieve its slow release, in order to maintain the necessary threshold concentration of the active ingredient more time on the soils and so reduce the pollution concentrations.

It is known that the MCPA can be effectively removed from water by coagulants, such as aluminum and ferric salts; ${ }^{[27,28]}$ and biochars. ${ }^{[29]}$ In order to attain a good clarification, the adsorbent must accomplish at least the following properties: size lower than $0.5 \mu \mathrm{m}$, which produce a high specific surface area and strong interactions to retain the contaminant. Properties that meet the OMMT samples, as poly(diallyldimethylammonium)-sepiolite, which has been used successfully to clarify winery effluent, diminishing the contact time used with clay and alum treatment from $24 \mathrm{~h}$ to less than $2 \mathrm{~min}^{\left[{ }^{[30]}\right.}$

The main objective of this work was to evaluate the MCPA adsorption capacity of raw MMT and three OMMT samples obtained by different percentages of dodecyl trimethyl ammonium cation loading. The samples were characterized by determination of total specific surface and apparent particle diameter and by FTIR and thermal analysis. The XRD and zeta potential determinations were used to point out the importance of inner and outer surface sites in the MCPA adsorption mechanism. Also, the coagulation efficiency of MCPA adsorbed on the samples studied was compared to that of a commercial activated carbon.

\section{Experimental}

\section{Materials}

A smectite sample collected from Lago Pellegrini deposit (Rio Negro, North Patagonia, Argentine) was provided by Castiglioni Pes y Cia., used as received and named MMT. The main properties of this clay determined previously ${ }^{[31]}$ are: CEC 174 $\mathrm{meq} / 100 \mathrm{~g}$, isoelectric point (IEP) at $\mathrm{pH}=2.7$, outer/external surface area (determined by $\mathrm{N}_{2}$ adsorption) $34.0 \mathrm{~m}^{2} \mathrm{~g}^{-1}$ and total specific surface area (determined by water vapor adsorp$\left.\operatorname{tion}^{[32]}\right) 621 \mathrm{~m}^{2} \mathrm{~g}^{-1}$. The sample contained 84, 12 and $4 \%$ of montmorillonite, quartz and feldspar, respectively. The chemical analysis, determined on a purified MMT sample, indicated a high charge (0.41 eq/formula unit) and a structural formula: [( $\left.\left.\mathrm{Si}_{3.89} \mathrm{Al}_{0.11}\right)\left(\mathrm{Al}_{1.43} \mathrm{Fe}_{0.26} \mathrm{Mg}_{0.30}\right)\right] \mathrm{M}^{+}{ }_{0.41}{ }^{[31]}$

Dodecyl trimethyl ammonium (DDTMA) bromide was purchased from Sigma Aldrich Ci. (St. Louis, MO, USA), purity $98 \%$ and soluble in water, and used as received. The molecular weight of DDTMA bromide is $308.34 \mathrm{~g} \mathrm{~mol}^{-1}$ and critical micelle concentration (CMC) is $15.18 \mathrm{mM}^{[33]}$ The MCPA as dimethylamine salt was purchased from Sigma Aldrich Ci., with $98 \%$ purity, $866 \mathrm{~g} \mathrm{~L}^{-1}$ solubility in water and was used as received. Dimensions of DDTMA and MCPA calculated using molecular modeling and geometry optimization tools for HyperChem (Hyperchem software v 7.0) and the chemical structure are shown in Figure 1. The dimensions of the counterion (dimethyl amine), calculated as for MCPA and DDTMA, are: $0.421 \mathrm{~nm}$ length and $0.277 \mathrm{~nm}$ width.

\section{Methods}

\section{Organo-montmorillonite preparation}

Fractions of MMT were modified with the organic cation through ion exchange reaction of 50, 100 and 200\% CEC and denoted as MMT0.5, MMT1 and MMT2, respectively. To attain these organo-clays (OMMTs), $6 \mathrm{~g}$ of MMT was first dispersed in $1 \mathrm{~L}$ of deionized water, to which the amount of DDTMA bromide to attain the CEC percentage chosen (50\% and $100 \%$ ) was slowly added. The reaction mixtures were stirred for $6 \mathrm{~h}$ at $60^{\circ} \mathrm{C}$. A fraction of all products were dried for zeta potential determination (indicated as not washed samples). Another fraction of all OMMT samples, to be used for MCPA adsorption and further characterization of the adsorption

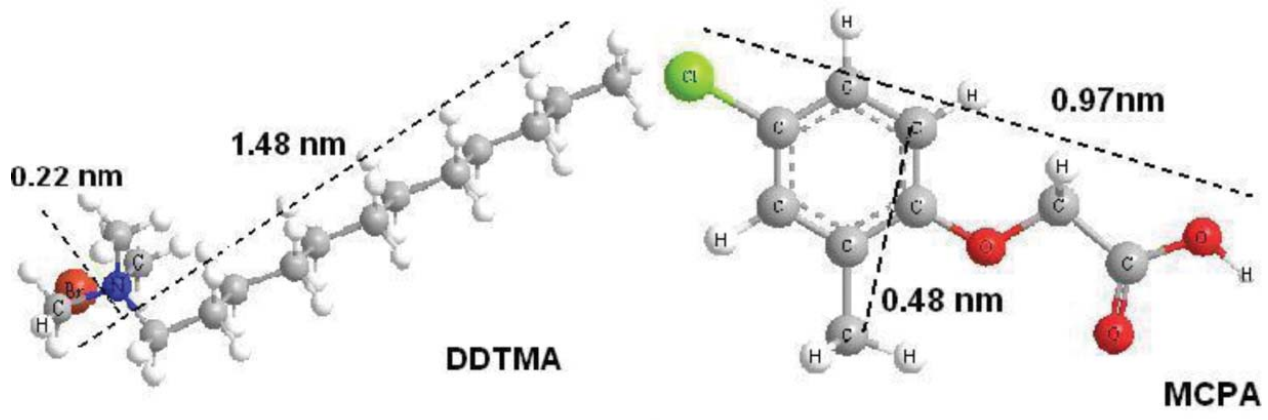

Figure 1. Chemical structure and dimensions of the indicated molecules. 
products, were washed by centrifugation to free them of bromide anions (tested by $\mathrm{AgNO}_{3}$ ), dried at $80^{\circ} \mathrm{C}$ and ground in an agate mortar The presence of $\mathrm{Br}^{-}$on washed OMMT samples was also controlled by wavelength dispersive spectrometry analysis, and a percentage lower than $0.03 \% \mathrm{Br}^{-}$was found in all samples.

\section{Adsorption experiments}

Before conducting the adsorption experiments, preliminary kinetic studies were carried out and $12 \mathrm{~h}$ was found to be long enough to reach pseudo-equilibrium (data not shown).

The adsorption of MCPA in MMT, MMT0.5, MMT1 and MMT2 samples was measured with a concentration range from 20 to $100 \mathrm{mg} \mathrm{MCPA} / \mathrm{L}\left(C_{i}\right)$ using the batch equilibration method. Duplicate suspensions $(1 \% \mathrm{w} / \mathrm{v})$ were equilibrated with several $\mathrm{mL}$ of MCPA at $\mathrm{pH} 6.5$, in order to attain the anionic form of MCPA (pKa: $3.07^{[11]}$ ) during the adsorption experiments, and shaken for $24 \mathrm{~h}$, at room temperature. After equilibration, the suspensions were centrifuged at $15,000 \mathrm{rpm}$ for $15 \mathrm{~min}$ and the amount of MCPA present in the supernatant was measured by UV spectrophotometry $(\lambda=230 \mathrm{~nm})$ using a Hewlett-Packard 8453 UV-visible spectrophotometer. All solutions and dispersions were prepared using MilliQ water. The MCPA adsorption amounts were determined as the difference between initial concentration and that of the supernatant in equilibrium. Solid samples with MCPA adsorption were air dried and stored in desiccators over silica gel, at room temperature, for use in further characterizations.

To determine a possible exchange of DDTMA by MCPA in OMMT surface samples, the DDTMA concentration was measured on the supernatant after MCPA $\left(C_{i}=100 \mathrm{ppm}\right)$ adsorption. To attain that, $0.01 \mathrm{~g}$ of MMT1-MCPA and MMT1 samples was dispersed in $10 \mathrm{~mL}$ water and shaken for $24 \mathrm{~h}$, at room temperature. Then the suspensions were centrifuged at $15,000 \mathrm{rpm}$ for $15 \mathrm{~min}$ and the amount of DDTMA present in the supernatant, in both samples, was measured by UV spectrophotometry $(\lambda=485 \mathrm{~nm}){ }^{[34]}$ No difference was found in DDTMA concentrations from both samples measured, which indicated the absence of DDTMA exchange by the presence of MCPA.

In this study, the obtained isotherms were fitted by several models (Langmuir, Freundlich, Dubinin-Radushkevich and Toth), using the SigmaPlot 10.0 software (Statistics - regression Wizard). Only Langmuir and Freundlich models presented the best fittings and consequently were chosen for the analysis. Although they were derived from the adsorption on solid-gas systems, they have been extensively applied to describe different sorption systems.

\section{Characterization methods}

The total specific surface area TSSA (Sw) was determined by water vapor adsorption at relative humidity (rh) 0.56, as described elsewhere. ${ }^{[35]}$ Inner or interlayer SSA was determined as the difference of TSSA and outer/external (ESSA). ${ }^{[32]}$ As adsorption induces swelling of the montmorillonite clay, TSSA determination by water adsorption may be used with the precaution of similar initial outgassing conditions. ${ }^{[24]}$
The apparent equivalent sphere diameter (Dapp) was obtained by dynamic light scattering DLS measurements with a Zeta Potential Analyzer 90Plus/Bi-MAS Multi Angle Particle Sizing (Brookhaven Instruments Corporation, Holtsville, NY, USA), operating at $\lambda=635 \mathrm{~nm}, 15 \mathrm{~mW}$ solid state laser, scattering angle $90^{\circ}, 25 \pm 0.1^{\circ} \mathrm{C}$. All sample suspensions $(1 \% \mathrm{w} / \mathrm{w})$ were prepared with water solutions and sonicated for $5 \mathrm{~min}$.

FTIR spectra of samples were recorded with a Perkin Elmer FTIR Spectrum One spectrometer (Perkin Elmer, Waltham, MA, USA), from $\mathrm{KBr}$ pellets.

TG/DTA experiments were carried out using a NETZSCH Simultaneous thermal analyzer (STA) 409 PC/PG, with alumina as reference (NETZSCH, Exton, PA, USA). The samples were placed into Pt crucibles and maintained at air throughout the heating period. The temperature was increased at a constant rate of $10^{\circ} \mathrm{C} \mathrm{min}^{-1}$.

Crystallographic data of samples with and without MCPA adsorbed were obtained by XRD on semioriented (powder) samples with a Philips 3710 diffractometer (the Netherlands) using $\mathrm{Cu} \mathrm{K}_{\alpha}$ radiation $40 \mathrm{kV}, 20 \mathrm{~mA}$, Ni filter and patterns collected from 3 to $12^{\circ}(2 \theta)$.

Zeta potential measurements were carried out using the same Brookhaven equipment (Zeta Potential function) as that utilized for Dapp measurements, at constant ionic strength of $10^{-3} \mathrm{M} \mathrm{KCl}$. Samples were prepared at several $\mathrm{pH}$ values equilibrated for $24 \mathrm{~h}$ by dropwise addition of concentrated $\mathrm{HCl}$ or $\mathrm{KOH}$ solutions.

\section{Coagulations process}

The flocculation efficiency of the adsorbents used after MCPA adsorbed was compared with a sample of commercial activated carbon. The coagulation efficiency was measured as a function of time, in suspensions of $0.1 \% \mathrm{w} / \mathrm{w}$ of each sorbent with addition of $50 \mathrm{mg} \mathrm{MCPA} \mathrm{L}^{-1}$ concentration at time 0 .

\section{Results and discussion}

\section{Sorption of MCPA}

The experimental isotherms of MCPA adsorption for the MMT and OMMTs samples were obtained with their corresponding Langmuir fits (Fig. 2). MCPA adsorption was greater for OMMT than for MMT sample, and an increase of MCPA with DDTMA loading increase was also found (Fig. 2).

The experimental data of MCPA adsorption on different OMMTs attained the best fitting $\left(R^{2}\right)$ with Langmuir and Freundlich models and the parameters related to both models are summarized in Table 1.

Although in the range of concentrations tested (Fig. 2) there were no significant differences between the MCPA adsorption in MMT0.5 and MMT1 samples, the fit of experimental data to Langmuir model showed an increase of the maximum adsorption amount $\left(q_{\max }\right)$ for these surfactant loadings (Table 1).

The best $q_{\max }$ value was found for the MMT2 sample (Table 1), being approximately $8.5,3.1$ and 1.8 times higher than that obtained for MMT, MMT0.5 and MMT1, respectively. These values were in between those reported for 


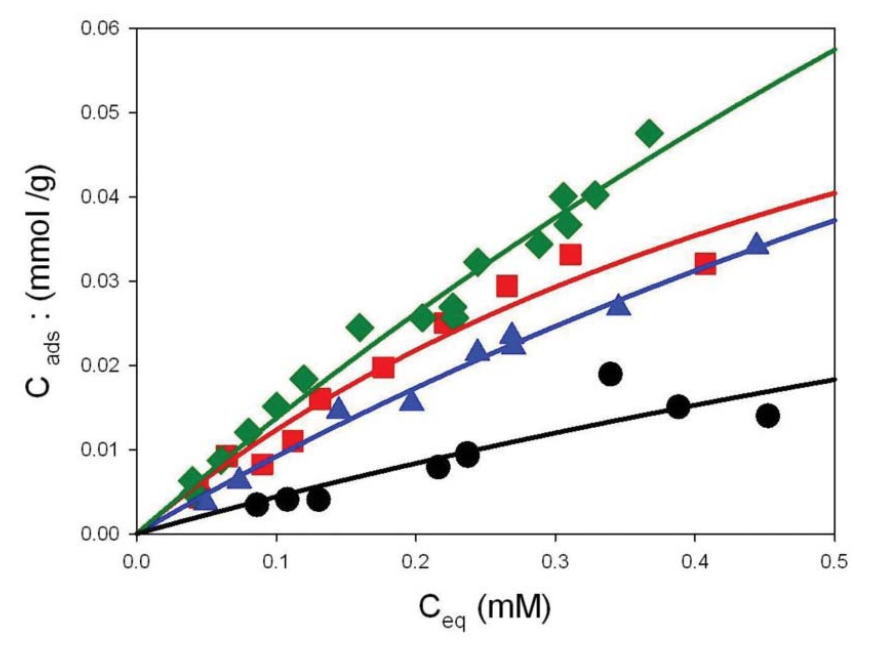

Figure 2. Adsorption isotherms of MCPA on MMT and OMMTs samples. Symbols indicate: (circle, white) MMT; (square, red) MMT0.5; (triangle, blue) MMT1, and (diamond, green) MMT2 samples. The symbols represent averages from two replicates and the maximum scatter was less than $\pm 5 \%$ of the average values. Solid lines were calculated using the Langmuir data from Table 1.

activated carbon $\left(1.94 \mathrm{mmol} \mathrm{g}^{-1[12]}\right.$ and for dodecyl-ammonium-bentonite $\left(0.06 \mu \mathrm{mol} \mathrm{g}^{-1[17]}\right)$.

Increased adsorption among samples seems to be related to the negative charge surface decrease (discussed later), which would indicate an adsorption mechanism related to the electrostatic attraction of MCPA with the adsorbents studied.

The differences among Langmuir adsorption constants $\left(k_{L}\right)$, with values close to 1.5 for MMT and MMT0.5 samples and around 0.5 for MMT1 and MMT2 samples, indicate the different affinity of MCPA for each surface.

It is important to note that for MMT0.5 and MMT1 samples the amount of DDTMA used reached $34 \%$ and $69 \%$ of the CMC value, respectively, while for MMT2 sample it exceeded 37\% the CMC valueat which micelles canbeformed. Themicellespresence, found by XRD spectra comparison of the DDTMA and MMT2 samples (Fig. A1), could also influence the higher amount of MCPA adsorbedonMMT2 sample. Thisbehaviorisinagreement with that found by Mishael et al. ${ }^{[36]}$ for the high amount of Sulfometuron adsorption when micelles of octadecyltrimethylammonium was firstlyadsorbed on montmorillonite.

It is also important to highligth a $\mathrm{pH}$ change found, of about $0.5 \mathrm{pH}$ units to high $\mathrm{pH}$, for all adsorption isotherms after equilibration time. These $\mathrm{pH}$ changes can be assigned to inner sphere complex formation between the MCPA anionic form and the available metallic or silicon centers of the clay surface by the following equation:

$$
\equiv \mathrm{SOH}+\mathrm{MCPA}^{-} \rightleftarrows \mathrm{D} \equiv \mathrm{SMCPA}+\mathrm{OH}^{-}
$$

Table 1. Sorption constants obtained from the Langmuir and Freundlich isotherms of MCPA.

\begin{tabular}{lcccccccc}
\hline & \multicolumn{4}{c}{ Langmuir } & & \multicolumn{3}{c}{ Freundlich } \\
\cline { 2 - 4 } \cline { 7 - 8 } Sample & $R^{2}$ & $k_{L}(1 / \mathrm{mmol})$ & $q_{\max }\left(\mathrm{mmol} \mathrm{g}^{-1}\right)$ & & $R^{2}$ & $K_{F}$ & \multirow{2}{*}{$n$} \\
\hline MMT & 0.9582 & 1.431 & 0.034 & & 0.9302 & 0.031 & 1.134 \\
MMT0.5 & 0.9568 & 1.503 & 0.094 & & 0.9401 & 0.075 & 1.261 \\
MMT1 & 0.9877 & 0.611 & 0.159 & & 0.9826 & 0.068 & 1.175 \\
MMT2 & 0.9768 & 0.508 & 0.284 & & 0.9756 & 0.108 & 1.136 \\
\hline
\end{tabular}

While for OMMT, lyophilic (aromatic ring-alkyl chain) forces, between organo-clay surfaces and the MCPA molecules $^{[23]}$ could be assigned to increase the $\mathrm{pH}$ (around $0.5 \mathrm{pH}$ ) by protonation of the MCPA carboxylic group.

Freundlich parameters, which take into account adsorption on heterogeneous surfaces, also showed $1 / \mathrm{n}$ values ranging from 0.79 to 0.88 (Table 1), indicating no significant difference among surface heterogeneity of the samples. However, the $k_{F}$ values obtained clearly show a difference in the adsorption capacities among MMT2 and the other samples.

\section{Surface characterization}

As was indicated previously, ${ }^{[37]}$ an accurate determination of the TSSA of swelling clay minerals, such as montmorillonite, cannot be attained using $\mathrm{N}_{2}$ as probe molecule. ${ }^{[33]}$ The use of $\mathrm{N}_{2}$ only allows determining the ESSA of the clay, ${ }^{[38]}$ which ranges between $5 \%$ and $10 \%$ of the TSSA. ${ }^{[39]}$ As the water molecules penetrate the interlayer space, they are adsorbed on the inner surface as well and thus determine the TSSA value.

TSSA values decrease with the amount of exchanged DDTMA (Table 2), while Dapp values increase indicating the formation of larger agglomerates or the increase of stacked sheets that form the tactoids. The formation of these large aggregates, allows their separation from the solution with 10 min of centrifugation at $3,000 \mathrm{rpm}$, while for the sample of MMT $10 \mathrm{~min} \times 15,000 \mathrm{rpm}$ must be applied to isolate the solid. MCPA adsorption significantly decreased TSSA values for all samples, with respect to the same samples without MCPA (Table 2). This behavior is consistent with the results of MCPA adsorption since it decreased the number of free surface sites to adsorb water molecules. The increase in Dapp value for the MMT sample with MCPA adsorption indicated the formation of larger agglomerates with respect to the MMT sample (Table 2); again the electrostatic attractions generated by adsorption could be responsible for this behavior. While among OMMT samples the adsorption of MCPA, does not increase Dapp values significantly.

\section{FTIR characterization}

For a better analysis of the FTIR spectra, they were divided into four regions: from 3700 to $3000 \mathrm{~cm}^{-1}, 3000$ to $2800 \mathrm{~cm}^{-1}$, 1700 to $1600 \mathrm{~cm}^{-1}$ and 1550 to $1400 \mathrm{~cm}^{-1}$.

The first region (Figure not shown) corresponds to the $\mathrm{OH}$ stretching region, and was evidenced by the presence of the two bands at $3700-3000 \mathrm{~cm}^{-1}$ found for MMT and OMMTs. ${ }^{[40]}$ Also the intense peak at $3630 \mathrm{~cm}^{-1}$ and the

Table 2. Total specific surface area (TSSA) and Dapp values of indicated samples.

\begin{tabular}{lccccc}
\hline & \multicolumn{2}{c}{ TSSA $\left(\mathrm{m}^{2} \mathrm{~g}^{-1}\right)$} & & \multicolumn{2}{c}{ Dapp $(\mathrm{nm})$} \\
\cline { 2 - 3 } \cline { 5 - 6 } Sample & Without MCPA & With MCPA & & Without MCPA & With MCPA \\
\hline MMT & $573 \pm 15$ & $83 \pm 9$ & & $540 \pm 30$ & $871 \pm 65$ \\
MMT0.5 & $196 \pm 12$ & $21 \pm 5$ & & $1459 \pm 99$ & $1320 \pm 83$ \\
MMT1 & $136 \pm 11$ & $24 \pm 6$ & & $2150 \pm 170$ & $1545 \pm 88$ \\
MMT2 & $108 \pm 11$ & $30 \pm 6$ & & $2349 \pm 170$ & $2443 \pm 193$ \\
\hline
\end{tabular}




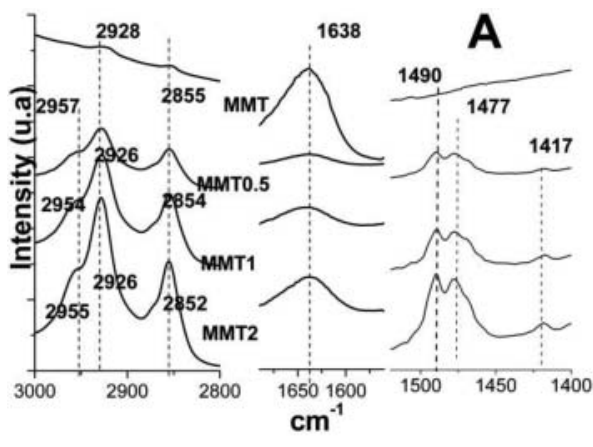

Figure 3. FTIR spectroscopy of MMT and OMMTs: (A) without and (B) with MCPA.

broad one at $3453 \mathrm{~cm}^{-1}$ found for MMT were assigned to $\mathrm{OH}$ stretching vibrations and to water molecules within the interlayer. The first one, in OMMT samples, was independent of the DDTMA concentration, while the second one was strongly dependent on DDTMA concentration and showed a shift to 3437,3433 and $3434 \mathrm{~cm}^{-1}$ for MMT0.5, MMT1 and MMT2, respectively, in agreement with that found for tetra- and hexa-decyltrimethyl-ammonium-MMT. ${ }^{[41]}$

In this region, MCPA adsorption on all samples does not notoriously change the peak shape and band position, despite the overlapping of the MCPA band (peak at $3448 \mathrm{~cm}^{-1}$ ) assigned to the intermolecular association $\mathrm{OH}$ as dimer, typically of acetic acid derivatives. ${ }^{[42]}$

The second region corresponds to the $\mathrm{CH}$ stretching region from $2900-2800 \mathrm{~cm}^{-1}$ (Fig. $3 \mathrm{~A}$ ), where the characteristic peaks at 2855 and $2928 \mathrm{~cm}^{-1}$ were assigned to symmetric and asymmetric $v\left(\mathrm{CH}_{2}\right)$ stretching modes of DDTMA, respectively. These peaks shifted to low wavenumber with the surfactant increase and were assigned to interlayer packing density. ${ }^{[20]}$ In Figure $3 \mathrm{~B}$, the FTIR spectra of added MCPA showed the characteristic peaks at 2963, 2927, 2907 and $2855 \mathrm{~cm}^{-1}$ assigned to CH stretching. ${ }^{[3]}$ The presence of MCPA was evidenced by the appearance of the peak at $2907 \mathrm{~cm}^{-1}$, only detected in MMT sample.

The third region characterizes the $\mathrm{HOH}$ bending vibrations of water molecules adsorbed on MMT and OMMTs, indicated by the peak at $1638 \mathrm{~cm}^{-1}$ that shifted to $1633 \mathrm{~cm}^{-1}$ and decreased its intensity with the surfactant increase (Fig. $3 \mathrm{~A}$ ). The adsorption of MCPA (Fig. 3 B) does not exhibit the band for carboxylic and carbonyl groups from MCPA, corresponding to bands at 1745 and $1710 \mathrm{~cm}^{-1}$.20]

In the region from 1550 to $1400 \mathrm{~cm}^{-1}$, the methylene scissoring mode at $1480-1440 \mathrm{~cm}^{-1}$ showed two bands at 1490 and $1477 \mathrm{~cm}^{-1}$ (Fig. $3 \mathrm{~A}$ ), which decreased with the DDTMA concentration, and were assigned to the interaction between contiguous $\mathrm{CH}_{2}$ groups of neighboring chains in an orthorhombic arrangement of alkyl chains, indicating a prominent change of the surfactant local environment. In Figure $3 \mathrm{~B}$, the bands of DDTMA overlapped with that of MCPA; only in the MMT sample the presence of a slight band at $1494 \mathrm{~cm}^{-1}$ could indicate some presence of MCPA.

Due to the superposition of the typical signals of the MMT in the region $1000-1200 \mathrm{~cm}^{-1}$, it is not possible to identify the $\mathrm{CN}$ band at $1000-1200 \mathrm{~cm}^{-1}$ characteristic of amines (Figure not shown).

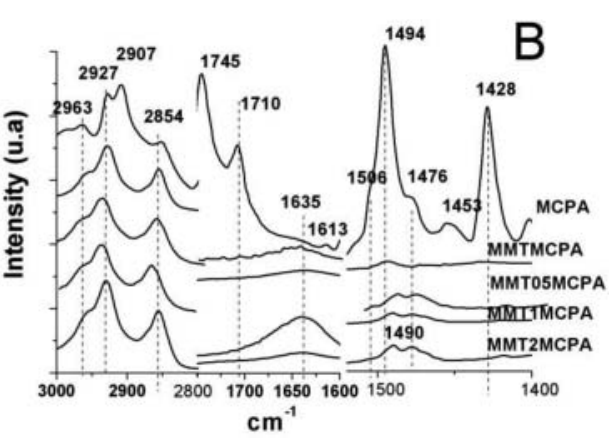

\section{Thermogravimetry (TG) characterization}

The weight loss of samples without and with MCPA (solids with maximum adsorption), was resumed in Table 3.

The classical two-step weight loss for the MMT sample $\left(0-200^{\circ} \mathrm{C}\right.$ and $\left.600-800^{\circ} \mathrm{C}\right)$ corresponded to the surface adsorbed and hydration water associated with inorganic cations, and dehydroxylation reaction (loss of structural hydroxyls) to clay minerals, respectively. ${ }^{[44]}$ The behavior of the MMT sample above $800^{\circ} \mathrm{C}$ was analogous to that found for other montmorillonites, corresponding to sintering. ${ }^{[4]}$

In the temperature range $0-200^{\circ} \mathrm{C}$, for the $\mathrm{MMT}$ sample (11.0\%) dehydration was greater than for OMMT samples (around $2.0 \%$ ), which is consistent with the higher hydration sphere formed by the MMT inorganic cations with respect to the organic cations (DDTMA) of the OMMTs. ${ }^{[16]}$ Between 200 and $800^{\circ} \mathrm{C}$, the presence of DDTMA increased the weight loss from $5.0 \%$ up to $16.4 \%$ for MMT and OMMT samples, respectively. This increase in weight loss is assigned to decomposition of DDTMA molecules, which need higher decomposition temperature $\left(194^{\circ} \mathrm{C}\right)$ when they are bonded on the clay surfaces. ${ }^{[45]}$

The presence of MCPA produced a decrease in weight loss of $1.1 \%$, between 0 and $200^{\circ} \mathrm{C}$, in MMT-MCPA sample respect to MMT sample, assigned to the MCPA degradation (melting point $114-118^{\circ} \mathrm{C}$ ) and to the displacement of interlayer inorganic cations and its associated water. While for OMMT-MCPA samples, at same temperature range, the MCPA degradation produced a weight loss difference of $0.3 \%$ up to $0.8 \%$. Besides, between 200 and $800^{\circ} \mathrm{C}$ the lower $\%$ of DDTMA molecules decomposition with MCPA increase could indicate some replacement of MCPA by DDTMA at the OMMT surface samples.

Table 3. Weight loss percentage for indicated samples.

\begin{tabular}{lcc}
\hline Sample & $0-200^{\circ} \mathrm{C}$ & $200-800^{\circ} \mathrm{C}$ \\
\hline MMT & 11.0 & 5.0 \\
MMT-MCPA & 9.9 & 4.3 \\
MMT0.5 & 2.0 & 16.4 \\
MMT0.5-MCPA & 2.3 & 14.6 \\
MMT1 & 1.3 & 16.4 \\
MMT1-MCPA & 2.1 & 14.9 \\
MMT2 & 1.7 & 16.3 \\
MMT2-MCPA & 2.4 & 15.4 \\
\hline
\end{tabular}




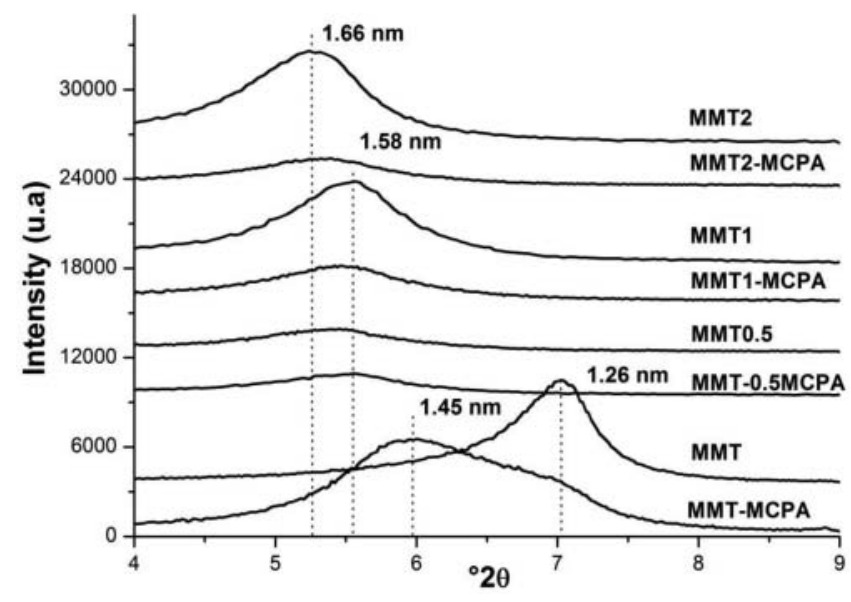

Figure 4. XRD patterns of indicated samples.

\section{Inner surface characterization (XRD)}

The XRD pattern of the MMT sample (Fig. 4) showed a reflection (001) peak at $1.26 \mathrm{~nm}\left(7^{\circ} 2 \theta\right)$ ratifying the predominance of a one-layer hydrate in the interlayer space. ${ }^{[46]}$ The adsorption of MCPA expanded the interlayer space $0.19 \mathrm{~nm}$ (to $1.45 \mathrm{~nm}$ ) for the MMT sample with a shoulder at $1.29 \mathrm{~nm}$, which implies the partial presence of a remnant monolayer arrangement in the interlayer space of the MMT-MCPA sample. In the MMT-MCPA sample, the deconvolution of the reflection (001) peak indicated a relationship area between the two peaks (1.45 and $1.29 \mathrm{~nm}$ ) of around 11.2, indicating the high predominance of MCPA interlayer entrance.

The shoulder observed at $1.29 \mathrm{~nm}$ could also be atributted to some interlayer entrance of the MCPA counterion (dimethyl amine), due to the well-known negative charge developed by MMT (discussed in further paragraphs) in a wide $\mathrm{pH}$ range. In order to confirm this behavior, the difference between the measured basal spacing observed upon the adsorption of MCPA and that of waterless $\mathrm{MMT}^{[46]}(1.45-0.96 \mathrm{~nm}=0.49 \mathrm{~nm})$ was determined to evaluate the interlayer available space. The estimated dimensions of the MCPA anion $(0.970 \times 0.480 \mathrm{~nm})$ and that of its counterion (dimethyl amine) $(0.421 \times$ $0.277 \mathrm{~nm}$ ) suggested that one orientation of both ionic forms could be responsible for the observed interlayer expansion involved in the MCPA adsorption mechanism. Particularly, the adsorption of the counterion (dimethyl amine) could be favored by the electrostatic attraction with the surface oxygen atoms of the tetrahedral layer of MMT, as found previously for

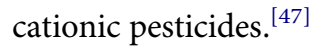

The analysis of the reflection (001) for the OMMT samples (from $50 \%$ to $200 \%$ CEC of exchanged DDTMA) indicated a shift of around $0.40 \mathrm{~nm}$ towards higher values with respect to the same reflection (001) found for the MMT sample, and attributed to DDTMA intercalation as a bilayer. ${ }^{[46]}$ The almost constant basal spacing (from 1.58 to $1.61 \mathrm{~nm}$ ) found for all OMMT indicated that with only $50 \%$ of DDTMA exchanged, the interlayer space reached its maximum expansion.

Upon adsorption of MCPA, the reflection (001) of OMMT samples evidenced a shift range between 0.05 and $0.08 \mathrm{~nm}$, towards higher values, with respect to the same reflection of respective samples without MCPA. These results suggested different associations of the organic ions, DDTMA and subsequent adsorption of MCPA in the clay interlayer.

a. The DDTMA presence in the interlayer space allowed slow or negligible adsorption of MCPA in the interlayer producing almost no change in the basal spacing. Consequently, the outer surface would mainly act as adsorbent to justify the adsorption values found (Fig. 2). The low shift of interlayer space $(0.05-0.08 \mathrm{~nm})$ obtained could also be produced by some entrance of the counterion, dimethyl amine, as could happen in the MMT sample.

b. MCPA entered the interlayer pushing out some DDTMA cations, modifying the initial arrangement of DDTMA.

The last hypothesis (b) was neglected because absence of DDTMA, within the experimental error, was found in the supernatant after adsorption of MCPA in MMT1-MCPA sample.

\section{Outer surface characterization (Zeta potential)}

The zeta potential values at $\mathrm{pH} 6.0$, for washed and not washed samples and those with two MCPA concentrations $\left(C_{i}=20\right.$ and $100 \mathrm{ppm}$ MCPA, respectively) was indicated in Figure 5.

The two different types of charges of the MMT surface (permanent negative charge on the face and changing from negative to positive with decreasing $\mathrm{pH}$ at the edge) determine its zeta potential. The MMT sample gave negative net charge in all range of $\mathrm{pH}$ values generated by the predominance of negative charges on the faces with respect to the positive charge on the edges of the particles. ${ }^{[47,48]}$

All OMMT samples (Fig. 5) showed a decrease of negative surface charge with respect to that found for the MMT sample and related directly with the amount of surfactant loading, attaining positive zeta potential values for $>100 \%$ surfactant loading. ${ }^{[9]}$ These behaviors reveal the adsorption of the surfactant on the outer surfaces, which neutralizes the initial negative charges of MMT. The inner surface (interlayer) charges do not participate in the charge behavior, because cationic exchange is the main chemical mechanism involved, without modifying the

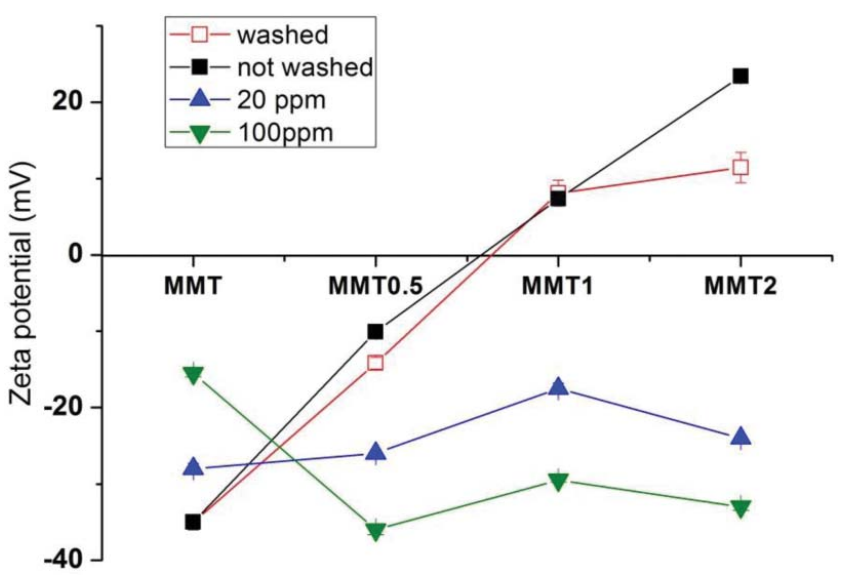

Figure 5. Zeta potential values at $\mathrm{pH} 6.0$ for indicated samples. Symbols indicate samples: ( $\square$ ) not washed, $(\square)$ washed and $(\boldsymbol{\Delta})$ with $20 \mathrm{mg}\left(C_{i}\right)$ and $(\boldsymbol{\nabla})$ with $100 \mathrm{mg}\left(C_{i}\right)$ MCPA. Lines located on the graph are only to assist view. $<$ PE $>$ Please note that here we have colored symbols. Please check for other instances also. 

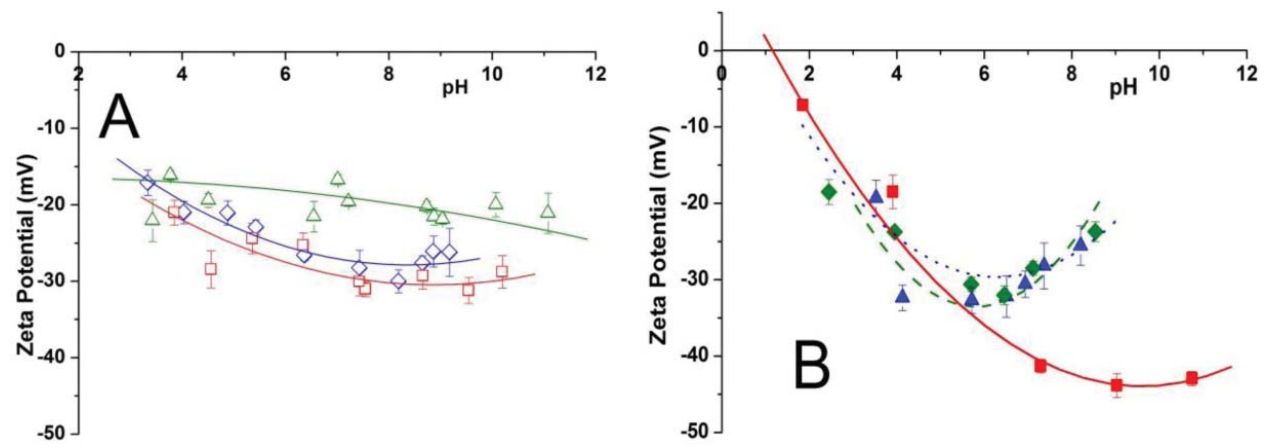

Figure 6. Zeta potential versus $\mathrm{pH}$ for indicated samples: $(\mathrm{A})$ with $C_{i}=20 \mathrm{ppm}$ and (B) with $C_{\max }$ MCPA. The fitting lines are a linear regression using a polynomial curve. Symbols indicate $(\boldsymbol{\square})$ MMT0.5; $(\boldsymbol{\Delta})$ MMT1 and $(\diamond)$ MMT2 samples. Lines located on the graph are only to assist viewing the curve taking into account the data dispersion.

inner surface charge, ${ }^{[48,50]}$ although as demonstrated by XRD measurements, the interlayer was almost completely exchanged with the lowest concentration of DDTMA (50\% CEC) used.

Water washing does not affect the MMT sample charge while for OMMT samples a linear relation of zeta potential values with the amount of surfactant loading was found. ${ }^{[51]}$ The water washing produced a high loss of positive charge only for MMT2 sample.

The MCPA adsorption in the MMT sample (Fig, 5) decreased the initial negative zeta potential $(-35 \mathrm{mV})$ in agreement with the increase of MCPA $\left(C_{i}=20\right.$ and $100 \mathrm{ppm}$ MCPA) to $-28 \mathrm{mV}$ and to $-15 \mathrm{mV}$, respectively. These losses of negative outer surface charge could be assigned to inner sphere complex formation between the MCPA anionic form and the available metallic or silicon centers of the clay surface, as was indicated by the increase of $\mathrm{pH}$ after equilibrium time in adsorptions of MCPA in all samples (Eq. 1). Some surface charge compensation by the cationic dimethyl amine counterion cannot be ruled, giving some consistence to assumption (a) discussed previously in section 3.4.

For the OMMT samples, it was found a direct increase of negative zeta potential with the $C_{i}$ of MCPA amount, without being evidenced the different amount of MCPA adsorbed for each OMMT samples (Fig. 2).

Evidence of a different behavior with MCPA adsorption among the OMMT samples can be observed by zeta potential curves at different $\mathrm{pH}$ (Fig. $6 \mathrm{~A}$ and B, for MCPA $C_{i}=20 \mathrm{ppm}$ and maximal amount adsorbed, respectively).

Particularly for the MMT0.5-MCPA sample, with maximum MCPA adsorption, the negative zeta potential values showed significant changes with the $\mathrm{pH}$ variation (Fig. $6 \mathrm{~B}$ ). The decrease of negative zeta potential at low $\mathrm{pH}$ (from -10 to $-20 \mathrm{mV}$ or lower $\mathrm{mV}$ ) was assigned to the protonation of MCPA $\left(\mathrm{pKa}=3.07^{[11]}\right)$ attached to the OMMT surface. The important increase of negative zeta potential values (from -25 to $-45 \mathrm{mV}$ ) observed at high $\mathrm{pH}$ could be assigned not only to the $\mathrm{pH}$ effect on the MCPA, but also to a higher dimethyl amine desorption on the edge surface, whose charge changed from positive to negative (IEP $\mathrm{pH}=7^{[48]}$ ).

For MMT1-MCPA and MMT2-MCPA samples the zeta potential value showed negative value similar to that found for the MMT0.5M-MCPA sample, evidencing a slight decrease and increase of negative surface charge with low and maximum MCPA adsorption, respectively, whose values were in between those found for MMT and MMT0.5 samples.

\section{Coagulation}

The coagulation performance of OMMT and commercial activated carbon samples was attained at $3 \mathrm{~h}$ (Fig. 7), while for MMT sample there was not attained a complete clarification of the solution at that time. These results were in agree with the higher agglomerates size determined by the Dapp measurements for OMMT respect to MMT sample (Table 1).

\section{Conclusions}

An adsorption increase of MCPA 3 times higher was found for OMMT samples with DDTMA than that found for MMT sample for the range of MCPA concentrations investigated. The different affinity behavior $\left(k_{L}\right)$ among MMT and MMT0.5,

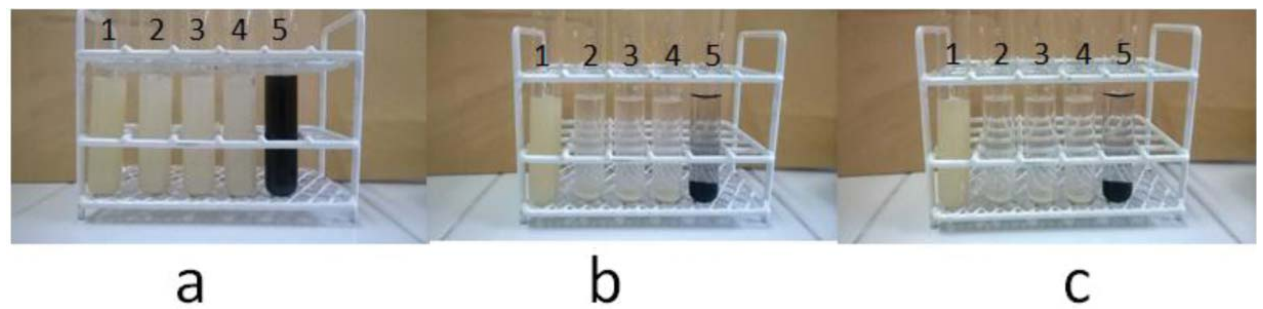

Figure 7. Addition of MCPA at time cero to: (1) MMT; (2) MMT0.5; (3) MMT1; (4) MMT2 and (5) commercial activated carbon samples. (a) 0 min; (b) 30 min; and (c) $180 \mathrm{~min}$. 
MMT1 and MMT2 samples, respectively, could be assigned to the formation of DDTMA micelles, mainly in MMT2 sample.

The analysis of XRD patterns and zeta potential curves from samples with and without different amounts of adsorbed MCPA indicated the presence of two adsorption sites. The first one was located at the interlayer, demonstrated by interlayer expansion with the increase in MCPA amount. The second one was located at the outer surface, evidenced by changes in the samples surface charge. Coagulation efficiency of OMMT with MCPA samples was better than that of MMT and similar to that obtained by commercial activated carbon. The high agglomerates formed by OMMT samples which remains after MCPA adsorption, allowed the remotion of water by a simple technological process.

\section{Funding}

The authors acknowledge the Secretaría de Ciencia y Técnica (ANPCyTFONARSEC) through Project FS-Nano 008. C.C.S. acknowledges a CONICET fellowship.

\section{References}

[1] Ignatowicz, K. Selection of sorbent for removing pesticides during water treatment. J. Hazard. Mater. 2009, 169, 953-957.

[2] Horvat, A.J.M.; Kaštelan Macan, M.; Petrović, M.; Barbarić, Ž. Study of MCPA and MCPP Herbicides Mobility in Soils from North-West Croatia as Affected by Presence of Fertilizers. J. Environ. Sci. and Health, Part B. 2003, 38(3), 305-316.

[3] Paszko, T. Adsorption and desorption processes of MCPA in Polish mineral soils. J. Environ. Sci. and Health, Part B. 2011, 46(7), 569580.

[4] Sørensen, S.R.; Schultz, A.; Jacobsen, O.S.; Aamand, J. Sorption desorption and mineralisation of the herbicides glyphosate and MCPA in samples from two Danish soil and subsurface profiles. Environ. Pollut. 2006, 141, 184-194.

[5] Jacobsen, C.S.; van der Keur, P.; Iversen B.V.; Rosenberg, P.; Barlebo, H.C.; Torp, S.; Vosgerau, H.; Juhler, R.K.; Ernstsen, V.; Rasmussen, J.; Brinch, U.C.; Jacobsen, O.H. Variation of MCPA, metribuzine, methyltriazine-amine and glyphosate degradation, sorption, mineralization and leaching in different soil horizons. Environ. Pollut. 2008, 156, 794-802.

[6] Paszko, T. Modeling of $\mathrm{pH}$-dependent adsorption and leaching of MCPA in profiles of Polish mineral soils. Sci. Total Environ. 2014, 494-495, 229-240.

[7] Ahmad, T.; Rafatullah, M.; Ghazali, A.; Sulaiman, O.; Hashim, R.; Ahmad, A. Removal of Pesticides from Water and Wastewater by Different Adsorbents: A Review. J. Environ. Sci. and Health, Part C. 2010, 28, 231-271.

[8] Bruna, F.; Celis, R.; Pavlovic, I.; Barriga, C.; Cornejo, J.; Ulibarri, M.A. Layered double hydroxides as adsorbents and carriers of the herbicide (4-chloro-2-methylphenoxy) acetic acid (MCPA): Systems $\mathrm{Mg}-\mathrm{Al}, \mathrm{Mg}-\mathrm{Fe}$ and $\mathrm{Mg}-\mathrm{Al}-\mathrm{Fe}$. J. Hazard. Mater. 2009, 168, $1476-$ 1481.

[9] Kamaraj, R.; Davidson, D. J.; Sozhan, G.; Vasudevan, S. An in situ electrosynthesis of metal hydroxides and their application foradsorption of 4-chloro-2-methylphenoxyacetic acid (MCPA) from aqueous solution. J. Environ. Chem. Eng. 2014, 2, 2068-2077.

[10] Addorisio, V.; Esposito, S.; Sannino, F. Sorption Capacity of Mesoporous Metal Oxides for the Removal of MCPA from Polluted Waters. J. Agric. Food Chem. 2010, 58, 5011-5016.

[11] Ding, L.; Lu, X.; Deng, H.; Zhang, X. Adsorptive Removal of 2,4Dichlorophenoxyacetic Acid (2,4-D) from Aqueous Solutions Using MIEX Resin. Ind. Eng. Chem. Res. 2012, 51, 11226-11235.
[12] Kim, T.Y.; Park, S.S.; Kim, S.-J.; Cho, S.-Y. Separation characteristics of some phenoxy herbicides from aqueous solution. Adsorption. 2008, 14, 611-619.

[13] De Martino, A.; Iorio, M.; Xing, B.; Capasso, R. Removal of MCPA from water by sorption on carbon nanotubes and metal oxide nanoparticles. RSC Advances. 2012, 2, 5693-5700.

[14] Ayar, N.; Bilgin, B.; Atun, G. Kinetics and equilibrium studies of the herbicide 2,4-dichlorophenoxyacetic acid adsorption on bituminous shale. Chem. Eng. J. 2008, 138, 239-248.

[15] Celis, R.; Koskinen, W.C.; Hermosin, M.C.; Ulibarri, M.A.; Cornejo, J. Triadimefon interactions with organoclays and organohydrotalcites. Soil Sci. Soc. Am. J. 2000, 64, 36-43.

[16] Celis, R.; Trigo, C.; Facenda, G.; Hermosín, M.C.; Cornejo, J. Selective Modification of Clay Minerals for the Adsorption of Herbicides Widely Used in Olive Groves. J. Agric. Food Chem. 2007, 55, 66506658.

[17] Akçay, G.; Akçay, M.; Yurdakoç, K. The characterization of prepared organomontmorillonite (DEDMAM) and sorption of phenoxyalkanoic acid herbicides from aqueous solution. J. Colloid Interface Sci. 2006, 296, 428-433.

[18] Sanchez-Martin, M.J.; Rodriguez-Cruz, M.S.; Andrades, M.S.; Sanchez-Camazano, M.J. Efficiency of different clay minerals modified with a cationic surfactant in the adsorption of pesticides: influence of clay type and pesticide hydrophobicity. Appl. Clay Sci. 2006, 31, 216-228.

[19] Olivelli, M.S.; Curutchet, G.A.; Torres Sánchez, R.M. Uranium Uptake by Montmorillonite-Biomass Complexes. Ind. Eng. Chem. Res. 2013, 52, 2273-2279.

[20] Zhu, J.; He, H.; Guo, J.; Yang, D.; Xie, X. Arrangement models of alkylammonium cations in the interlayer of $\mathrm{HDTMA}^{+}$pillared montmorillonites. Chin. Sci. Bull. 2003, 48, 368-372.

[21] Fielding, M.; Barcelo, D.; Helweg, A.; Galassi, S.; Torstensson, L.; Zoonen, P.; Wolte, R.; Angeletti, G. Pesticides in ground and drinking water. Water Poll. Res. Report 1992, 27, 1-136.

[22] US EPA. Available at http://archive.epa.gov/pesticides/reregistration/ web/pdf/mcpa_red_fs.pdf (accessed Dec 2015).

[23] Akçay, G.; Yurdakoç, K. Removal of various phenoxyalkanoic acid herbicides from water by organo-clays. Acta Hydroch. Hydrob. 2000, 28, 300-304.

[24] Torres Sánchez, R.M.; Genet, M.J.; Gaigneaux, E.M.; dos Santos Afonso, M.; Yunes, S. Benzimidazole adsorption on the external and interlayer surfaces of raw and treated montmorillonite. Appl. Clay Sci. 2011, 53, 366-373.

[25] Khoury, G.A.; Gehris, T.C.; Tribe, L.; Torres Sánchez, R.M.; dos Santos Afonso, M. Glyphosate adsorption on montmorillonite: An experimental and theoretical study of surface complexes. Appl. Clay Sci. 2010, 50, 167-175.

[26] Zhu, J.; Wang, T.; Zhu, R.; Ge, F.; Yuan, P.; He, H. Expansion characteristics of organo montmorillonites during the intercalation, aging, drying and rehydration processes: Effect of surfactant/CEC ratio. Colloids Surf. A 2011, 384, 401-404.

[27] Unuabonah, E. I.; Taubert. A. Clay-polymer nanocomposites (CPNs): Adsorbents of the future for water treatment. Appl. Clay Sci. 2014, 99, 83-92.

[28] Kamaraj, R.; Davidson, D. J.; Sozhan, G.; Vasudevan, S. An in situ electrosynthesis of metal hydroxides and their application for adsorption of 4-chloro-2-methylphenoxyacetic acid (MCPA) from aqueous solution. J. Environ. Chem. Eng. 2014, 2, 2068-2077.

[29] Sarijo, S. H.; Izaddin, S. A.; Ghazali, S.M.; Hussein, M. Z.; Sidek, N. J. Synthesis of nanocomposite 2-methyl-4-chlorophenoxyacetic acid with layered double hydroxide: physicochemical characterization and controlled release properties. J. Nanoparticle Res. 2013, 15, 1356.

[30] Trigo, C.; Spokas, K.A.; Cox, L.; Koskinen, W. C. Influence of Soil Biochar Aging on Sorption of the Herbicides MCPA, Nicosulfuron, Terbuthylazine, Indaziflam, and Fluoroethyldiaminotriazine. J. Agric. Food Chem. 2014, 62, 10855-10860.

[31] Magnoli, A.P.; Tallone, L.; Rosa, C.A.R.; Dalcero, A.M. Chiacchiera, S.M.; Torres Sánchez, R.M. Commercial bentonites as detoxifier of broiler feed contaminated with aflatoxin. Appl. Clay Sci. 2008, 40, 63-71. 
[32] Michot, L.J.; Villieras, F. in Developments in Clay Sci. Surface area and porosity. Handbook of Clay Science, Elsevier: Amsterdam, The Netherlands, 2006 p. 965.

[33] Xing, H.; Yan, P.; Zhao, K.; Xiao, J. Effect of Headgroup Size on the Thermodynamic Properties of Micellization of Dodecyltrialkylammonium Bromides. J. Chem. Eng. Data 2011, 56, 865-873.

[34] Few A.V.; Ottewill, R.H. A spectrophotometric method for the determination of cationic detergents. J. Colloid Sci. 1956, 11, 34-38.

[35] Torres Sánchez, R.M.; Falasca, S. Specific surface and surface charges of some Argentinian soils. J. Plant Nutr. Soil Sci. 1997, 160, 223-226.

[36] Mishael, Y.G.; Undabeytia, T.; Rytwo, G.; Papahadjopoulos-Sternberg, B.; Rubin, B.; Nir, S. Sulfometuron Incorporation in Cationic Micelles Adsorbed on Montmorillonite. J. Agric. Food Chem. 2002, 50, 2856-2863.

[37] Fernández, M.; Alba, M.D.; Torres Sánchez, R.M. Effects of thermal and mechanical treatments on montmorillonite homoionized with mono- and polyvalent cations: Insight into the surface and structural changes. Colloids and Surf. A. 2013, 423, 1-10.

[38] Caglar, B.; Afsin, B.; Tabak, A.; Eren, E. Characterization of the cation-exchanged bentonites by XRPD, ATR, DTA/TG analyses and BET measurement. Chem. Eng. J. 2009, 149, 242-248.

[39] Laird, D.A. Layer charge influences on the hydration of expandable 2:1 phyllosilicates. Clays Clay Miner. 1999, 47, 630-636.

[40] Ma, Y.; Zhu, J.; He, H.; Yuan, P.; Shen, W.; Liu, D. Infrared investigation of organo-montmorillonites prepared from different surfactants. Spectrochim. Acta A. 2010, 76, 122-129.

[41] Park, Y.A.; Godwin, A.; Frost, R.L. In Thermal analysis and application of organoclays for water purification. Churchman, G, Cresswell, R, \& Singh, B (Eds.) Proceedings of the combined Australian Regolith Geoscientists Association and Australian Clay Minerals Society Conference, Mildura, Victoria, 2012 p. 79-82.
[42] Silverstein, R.W.; Bassler, G.C. Spectrometric identification of organic compounds. J. Chem. Educ. 1962, 39(11), 546-553.

[43] Bellamy, L.J. in The Infrared Spectra of Complex Molecules, third ed., Chapman and Hall: London, 1975.

[44] Rouquerol, J.; Rouquerol, P.L.; Llewellyn, P.L. In Developments in Clay Sci. Surface area and porosity, Handbook of Clay Science, Elsevier: Amsterdam, The Netherlands, 2006, p 1003.

[45] Park, Y.; Ayoko, G.A.; Frost, R.L. Characterization of organoclays and adsorption of p-nitrophenol: Environmental application. J. Colloid Interface Sci. 2011, 360, 440-456.

[46] Lagaly, G.; Ogawa, M.; Dékámy, I. In Clay mineral organic interactions, Handbook of clay science. Elsevier: Amsterdam, The Netherlands, 2006, p 309.

[47] Lombardi, B.; Torres Sánchez, R.M.; Eloy, P.; Genet, M. Interaction of thiabendazole and benzimidazole with montmorillonite. Appl. Clay Sci. 2006, 33, 59-65.

[48] Thomas, F., Michot, L.J.; Vantelon, D.; Montarges, E., Prelot, B., Cruchaudet, M.J., Delon, F. Layer charge and electrophoretic mobility of smectites, Colloids and Surf. A, 1999, 159, 351-358.

[49] Bianchi, A.E.; Fernández, M.; Pantanetti, M.; Viña, R.; Torriani, I.; Torres Sánchez, R.M.; Punte, R. ODTMA and HDTMA Organomontmorillonites characterization: new insight by WAXS, SAXS and surface charge. Appl. Clay Sci., 2013, 83-84, 280-285.

[50] Chakir Bessiere, A.J.; Kacemia, K. E., Marouf, B. A comparative study of the removal of trivalent chromium from aqueous solutions by bentonite and expanded perlite. J. Hazard. Mater. 2002, B95, 29-46.

[51] Zadaka, D.; Radian, A.; Mishael, Y.G.; Applying zeta potential measurements to characterize the adsorption on montmorillonite of organic cations as monomers, micelles, or polymers. J. Colloid Interface Sci. 2010, 352(1), 171-177.

\section{Appendix}

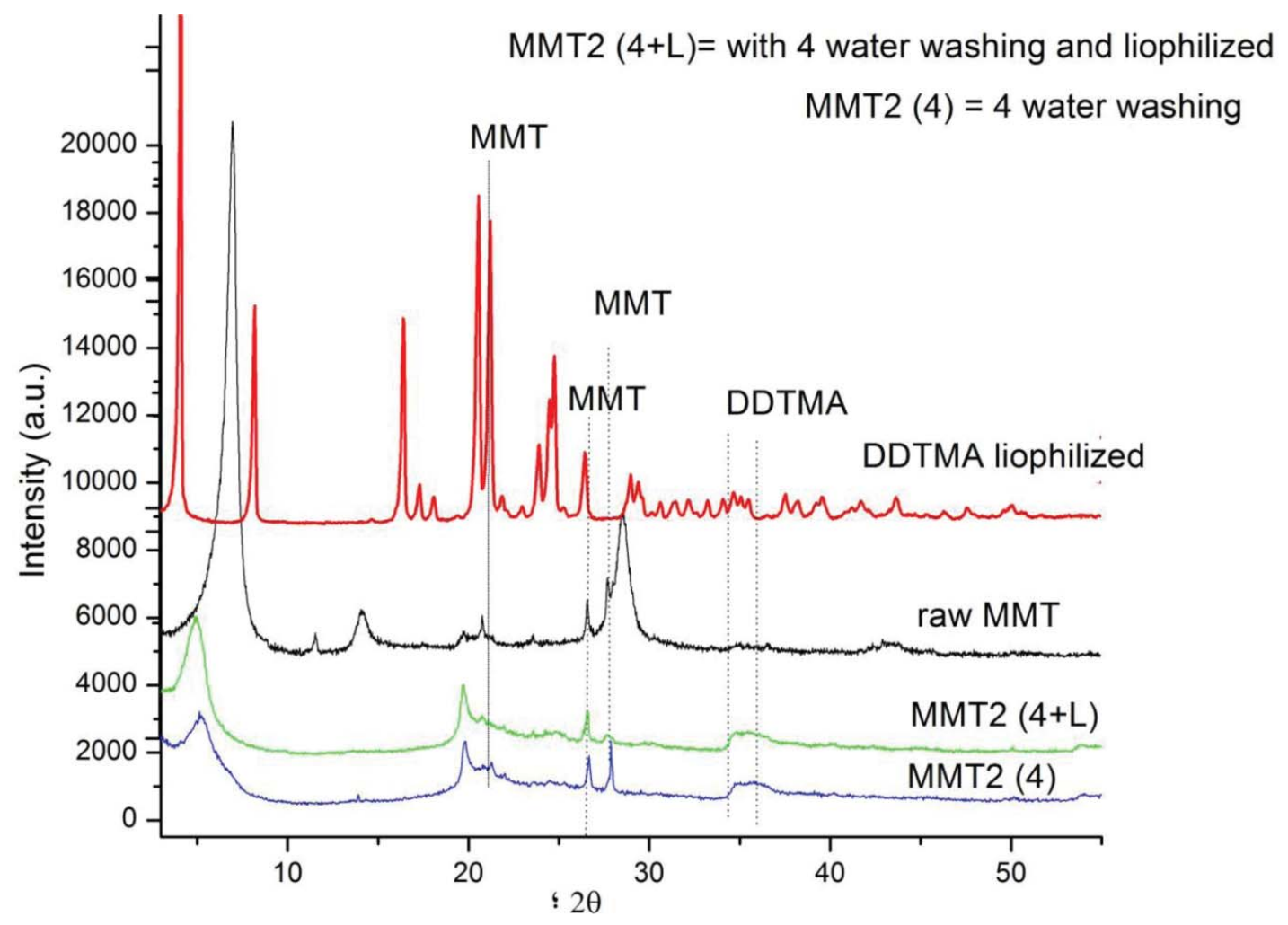

Figure A1. XRD of DDTMA lyophilized (freeze dryed), Rw MMT and MMT2 sample with 4 times water washing and lyophilized and with 4 times water washing. 